\title{
La prévention du risque d'inondation en Tarn-et-Garonne
}

\author{
Flood risk prevention in Tarn et Garonne
}

\author{
par Michèle Bordaries
}

DDE 82

In Tarn et Garonne, department crossed by the rivers Garonne, Tarn, Aveyron and their numerous tributaries, the government set up an ambitious policy, on three years time, to regain the expansion fields of the floods.

By the end of 1999, 194 «communes of flooding.

In 76000 acres, that is to say $10 \%$ of the department superficy, new constructions will be forbidden, except those linked with agricultural activities or for the river purpose.

\section{IINTRODUCTION}

En décembre 1996, les inondations du Tarn ont eu des conséquences très dommageables pour des dizaines d'entreprises commerciales ou industrielles de Sapiac et des alentours. Montauban et ses bas quartiers ont été dévastés, 600 maisons sinistrées. Une première estimation des dégâts montait à 135 millions de francs.

C'est le 19 décembre 1996, soit 11 jours après les crues catastrophiques du Tarn des 7 et 8 décembre 1996 que l'Etat annonce devant les maires réunis en Assemblée Générale, le contenu de sa nouvelle politique de construction en zone inondable.

Elle montre une politique ambitieuse de reconquête des champs d'expansion des crues, conduite sans relâche pen-

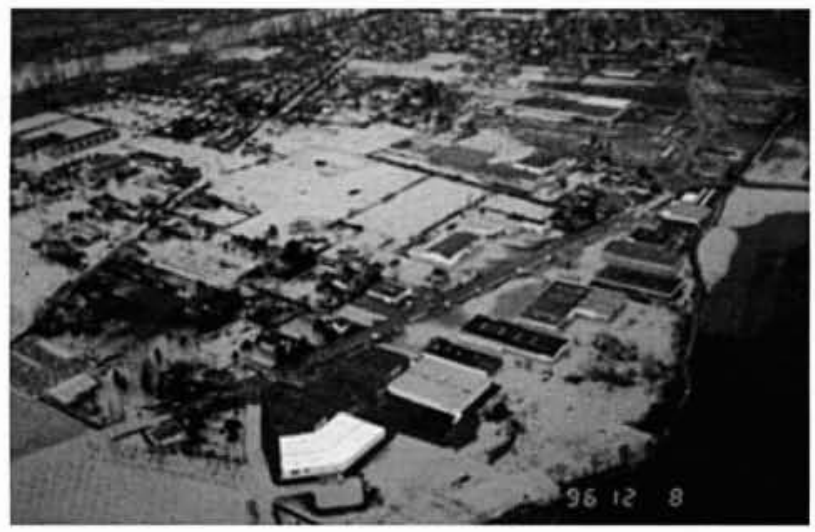

Crue du Tarn à Montauban - 1996. dant trois ans, dans un département coutumier des crues et des documents réglementaires qui se sont succédés depuis les plans de surface submersible.

Cette politique montre le chemin du bon sens puisque fin 1999:

- 194 communes sur 195 seront couvertes par un plan de prévention du risque inondation,

- 38000 hectares, soit $10 \%$ de la superficie du département seront réservés aux champs d'expansion des crues.

Elle aura nécessité de nombreuses réunions, explications, interventions pour que les communes comprennent l'intérêt d'inscrire leur développement dans le respect de l'habitat existant et dans un environnement de qualité.

Mais parlons du chemin parcouru.

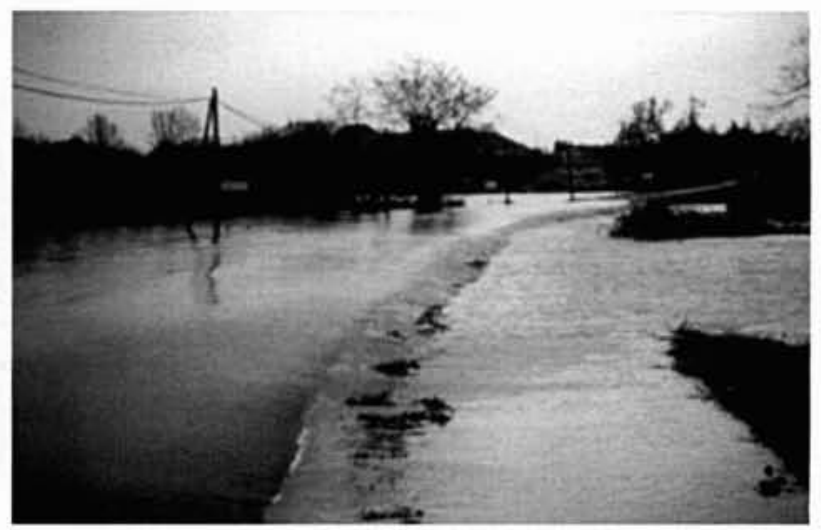

Crue du Tarn - 1996. 


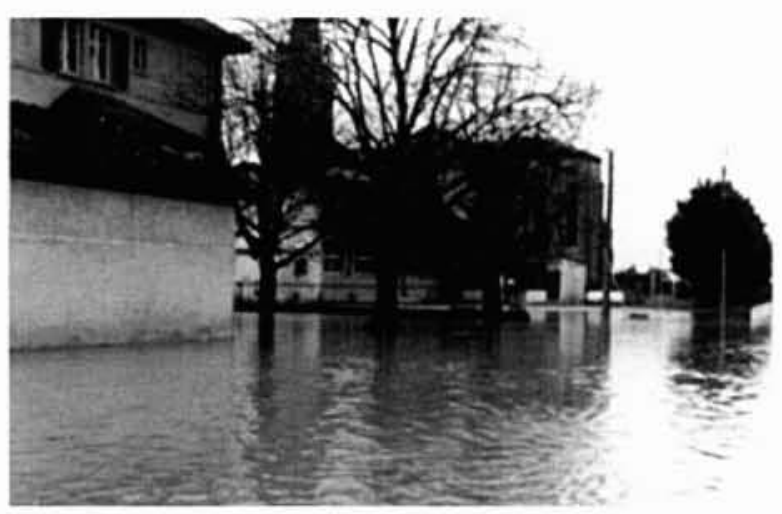

Crue du Tarn à Lizac - 1996.

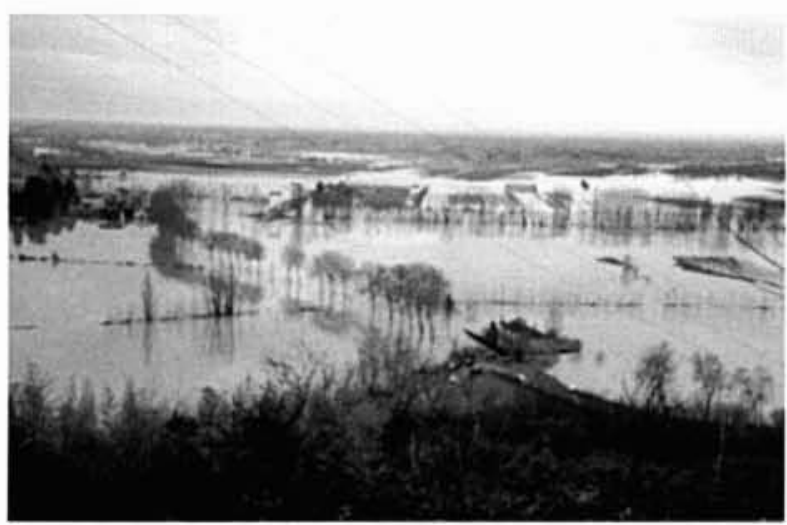

Crue du Tarn au Saula - 1996.

\section{II — L'ÉTAT DES LIEUX FIN 1996}

Le Tarn-et-Garonne est traversé par trois grands cours d'eau : la Garonne, le Tarn et l'Aveyron et de nombreux affluents.

La Garonne, le Tarn, l'Aveyron et le Viaur sont couverts par un réseau d'annonce des crues performant assuré par la DDE de Tarn-et-Garonne, et la Gimone et l'Arrats par un service d'annonce des crues assuré par la DDE du Gers.

Des crues historiques sont restées dans toutes les mémoires de par leur ampleur et l'importance des dégâts qu'elles ont provoqués :

- 1930, crue la plus forte connue pour le Tarn et pour l'Aveyron (112 morts à Moissac...).

- 1952 pour la Garonne (crue historique : 1875)

- 1981 pour l’Aveyron.

- 1982 pour le Tarn.

- 1996 pour le Tarn.

Le département de Tarn-et-Garonne a été pilote en matière de méthodologie de cartographie des risques inondation, ce qui a eu pour conséquence une couverture importante des vallées par des études spécifiques et l'élaboration d'un nombre conséquent de documents opposables, comme en témoigne la carte des documents de protection opposables en 1996.

Cependant les constats que l'on pouvait faire fin 96 étaient de trois ordres

\subsection{Des communes sans cartographie du risque}

Sur les 194 communes du département touchées par le risque d'inondation, seulement 70 étaient couvertes par un document opposable.

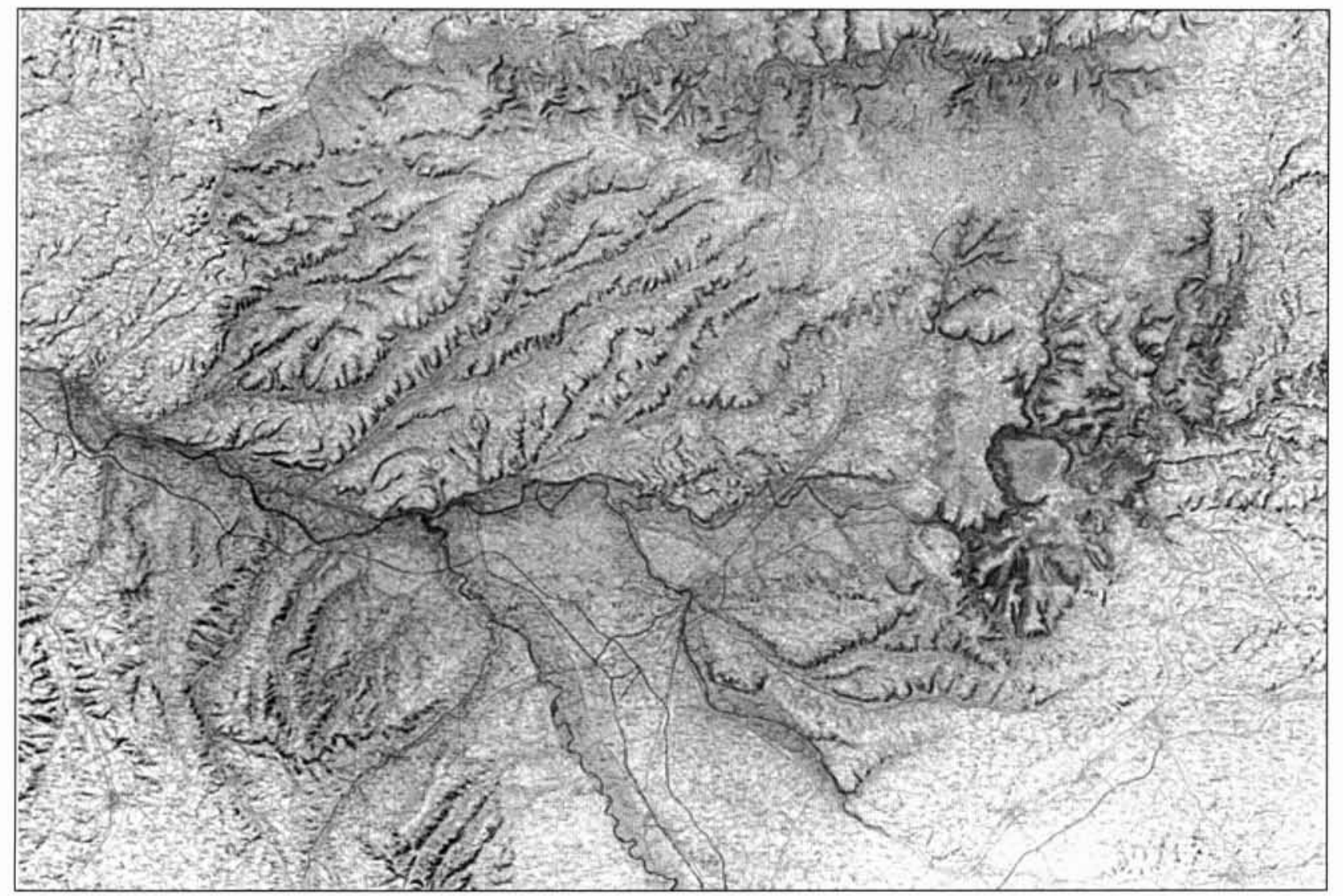

1. Cartographie des bassins du Tarn-et-Garonne. 


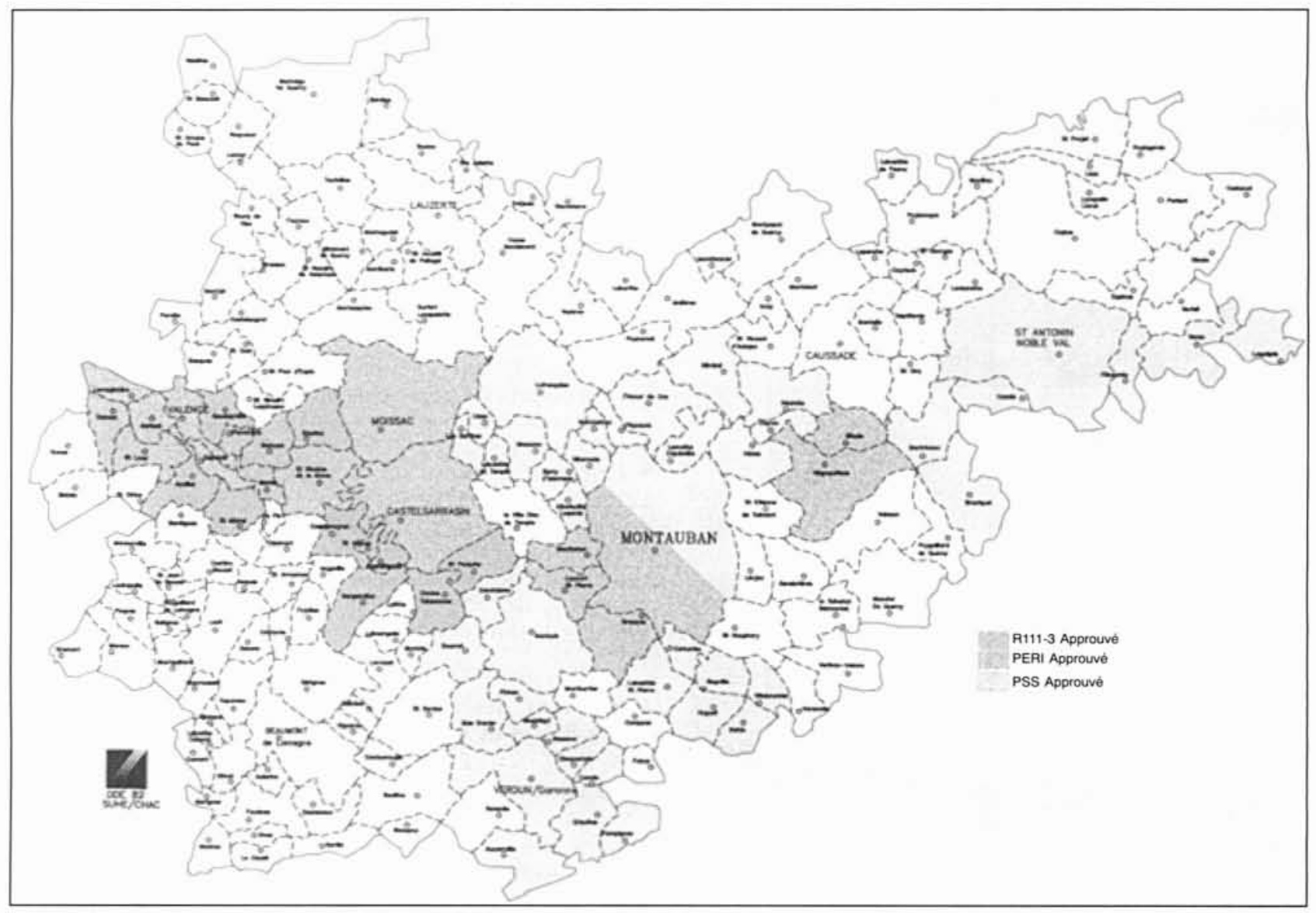

2. Prise en compte du risque d'inondation dans les documents d'urbanismes réglementaires.

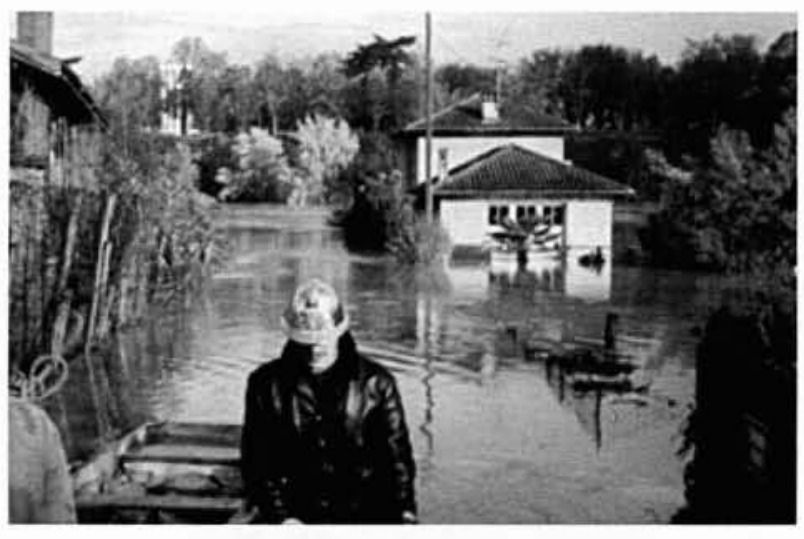

Crue du Tarn à Montauban - 1982.

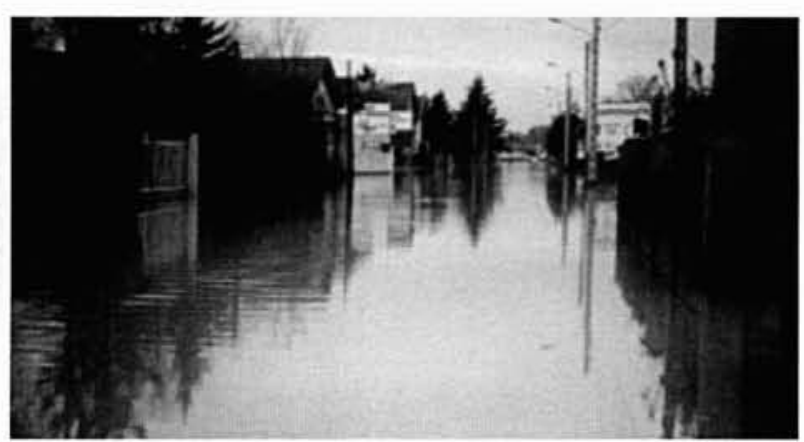

Crue du Tarn à Montauban - 1996.
- 1 P.S.S. couvrant 42 communes,

- 4. R 111.3 couvrant 15 communes,

- 13 communes couvertes par un Plan d'Exposition au Risque Inondation.

Sur 124 communes, le risque inondation était donc régi, au coup par coup, par les articles généraux du Code de l'Urbanisme traitant de la sécurité publique.

\subsection{Des références différentes}

Selon les documents élaborés, les critères de référence pris en compte variaient :

- crue historique pour les Plans de Surface submersible, centennale pour les autres documents ;

- hauteur de crue et vitesses de courants différents selon les vallées, voire même selon les communes;

- des études techniques parfois très lourdes;

- 2.3 des constructions en zone inondable, notamment autour des grandes agglomérations 


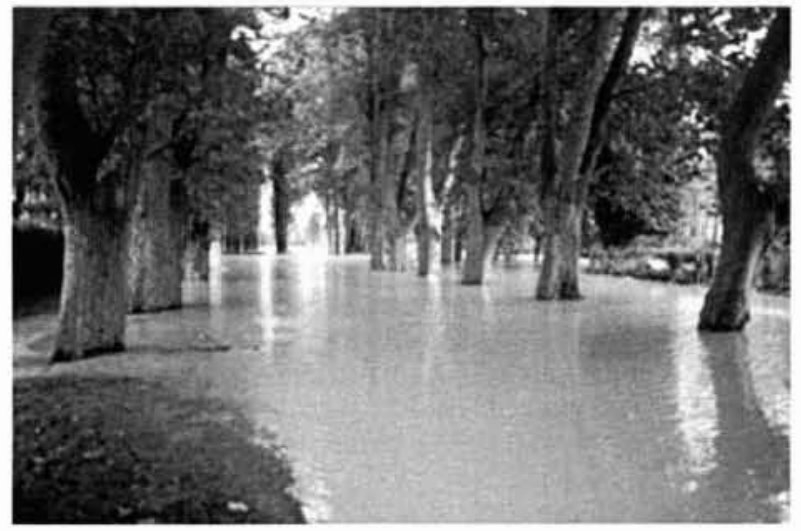

Crue de la Garonne - 1992.

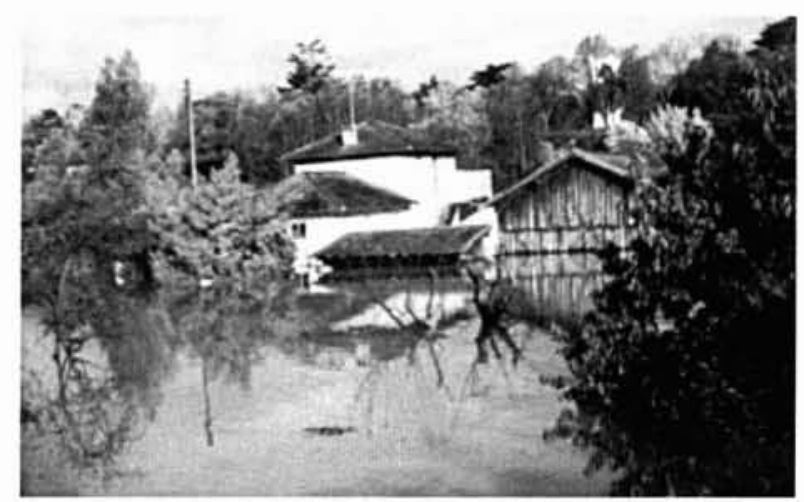

Crue du Tarn -1982.

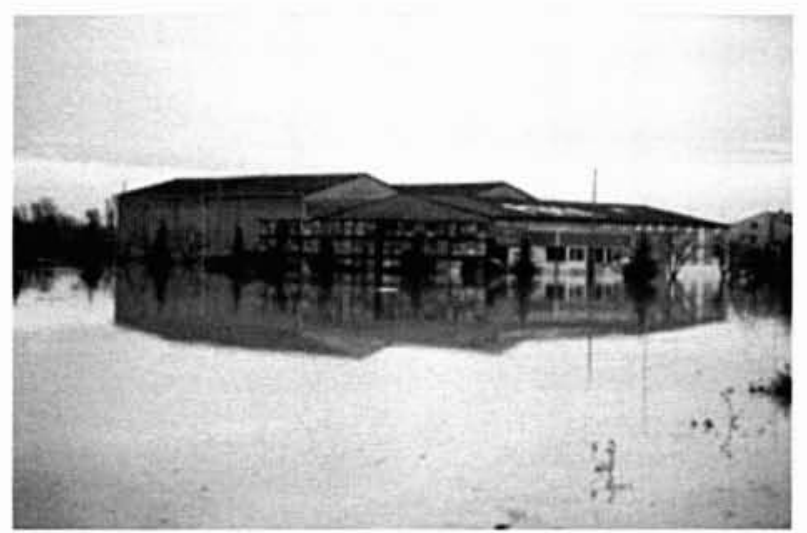

Crue du Tarn - 1996.

III —QUELS ENSEIGNEMENTS TIRER DE LA NOUVELLE POLITIQUE NATIONALE?

3.1 La loi du 2 février 1995 relative au renforcement de la protection de l'environnement et ses textes d'applications

C'est une nouvelle approche des risques naturels qui renverse les conceptions antérieures :

- La crue de référence à prendre en compte est la crue la plus forte connue,

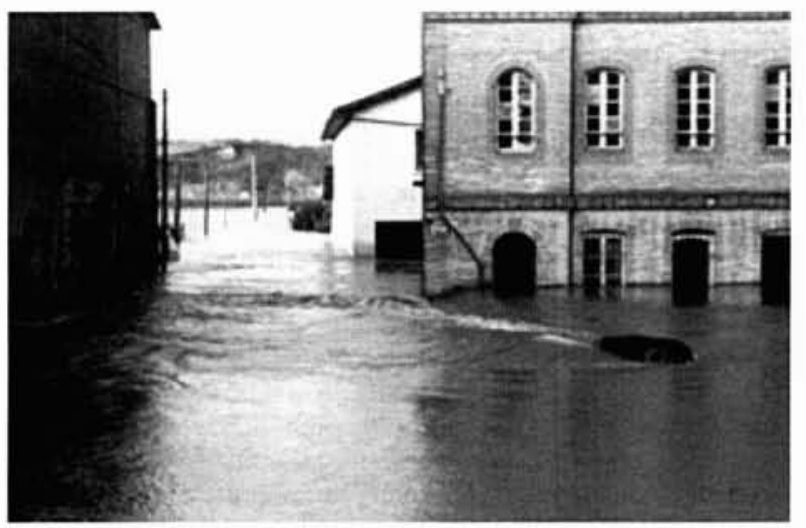

Crue de l'Aveyron - 1981.

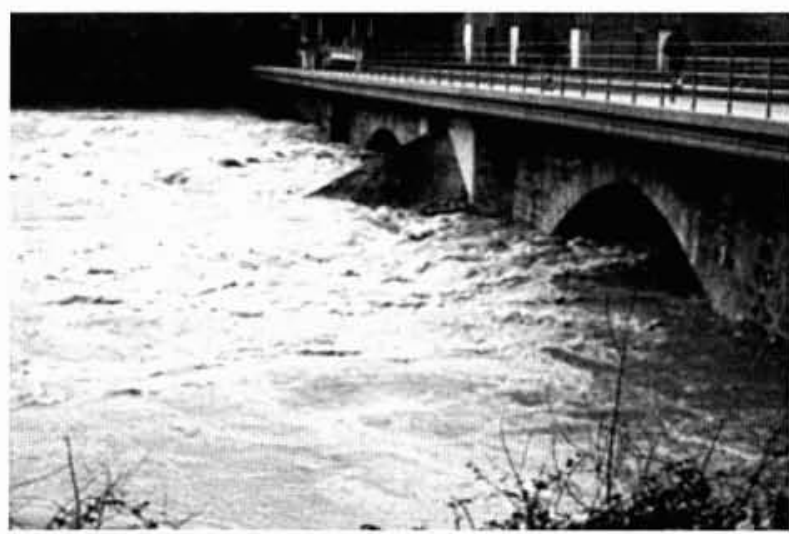

Crue de l'Aveyron - 1981.

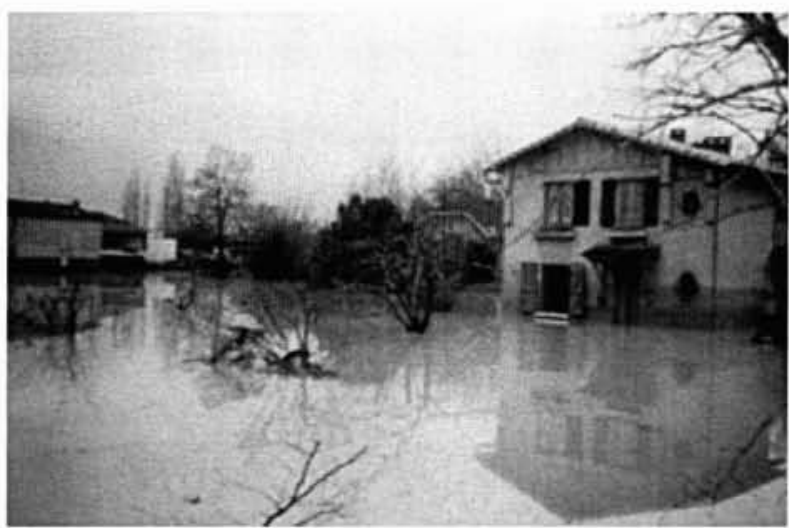

Crue du Tarn - 1996.

- En matière d'inondation, le lit majeur (zone couverte par la plus forte crue connue) devient inconstructible, l'objectif étant de préserver complètement les champs d'écoulement et de stockage des crues,

- Des adaptations sont possibles pour les zones fortement urbanisées et pour les zones déjà protégées par des digues.

\subsection{Les principes retenus en Tarn-et-Garonne}

- La plus forte crue connue à prendre en considération est la crue historique (c'est déjà une révolution).

- Pour les vallées non couvertes par un système d'annonce des crues : la totalité des zones inondables non couvertes par 
un système d'annonce des crues seront classées inconstructibles (Rouge).

- Dans les autres vallées :

Création de deux zones distinctes en zones inondables : bleu (B), rouge (R).

- La zone bleue est une zone urbanisée de faible submersion pour la crue de référence (la plus forte crue connue) avec des hauteurs de submersion $\leq 1 \mathrm{~m}$ et des vitesses d'écoulement $\leq 0,5 \mathrm{~m} / \mathrm{s}$, et couverte par un système d'annonce des crues, dans laquelle il est possible à l'aide de prescriptions de préserver les biens et les personnes.

- La zone rouge est une zone où les hauteurs ou les vitesses de submersions sont telles que la sécurité des biens et des personnes ne peut pas être garantie quels que soient les aménagements qui pourraient être apportés.

Sont également classées en zone Rouge, la totalité des zones submersibles non couvertes par un service d'annonce des crues et les zones submersibles non urbanisées (c'est l'essentiel pour la préservation des champs d'expansion des crues).

- Des adaptations pour les secteurs protégés et les centres anciens :

- Dans les zones de forte submersion pour la crue de référence, protégées par une digue contre la crue centennale, et couvertes par un système d'annonce des crues ( 2 zones seulement remplissent ces conditions).

- Les centres urbains denses, en zone de forte submersion sont soumis à la réglementation de la zone rouge avec des adaptations permettant aux quartiers de continuer à vivre sans augmenter les populations soumises à risque.

\subsection{La mise en œuvre des principes retenus en Tarn-et- Garonne}

Principes : la même règle pour toutes les communes élaborée dans la transparence :

- Délimitation par Arrêté Préfectoral du 9 juin 1997 de 4 secteurs d'étude couvrant l'ensemble du département, qui prennent en compte le cours d'eau principal et ses affluents, c'est-à-dire le sous-bassin et qui permettent de faire s'exprimer les solidarités Amont-Aval.

- Application anticipée des futures prescriptions des P.P.R. pour gérer la période transitoire.

- A partir d'avril 1997, à l'aide de l'article R 111-2 du Code de l'Urbanisme.

- Le $1^{\text {er }}$ octobre 1998 application anticipée des P.P.R. Garonne Amont et Aval.

- Le 18 décembre 1998 application anticipée du P.P.R. Tarn.

- Approbation du P.P.R. Aveyron couvrant 44 communes le 22 juin 1998.

- Approbation du P.P.R. Garonne Amont couvrant 54 communes le 19 juillet 1999.

\subsection{Résultats}

Ce dispositif préserve les champs d'expansion des crues (38 000 ha soit $10 \%$ du territoire) et interdit toute construction dans les zones urbaines où l'aléa est fort.

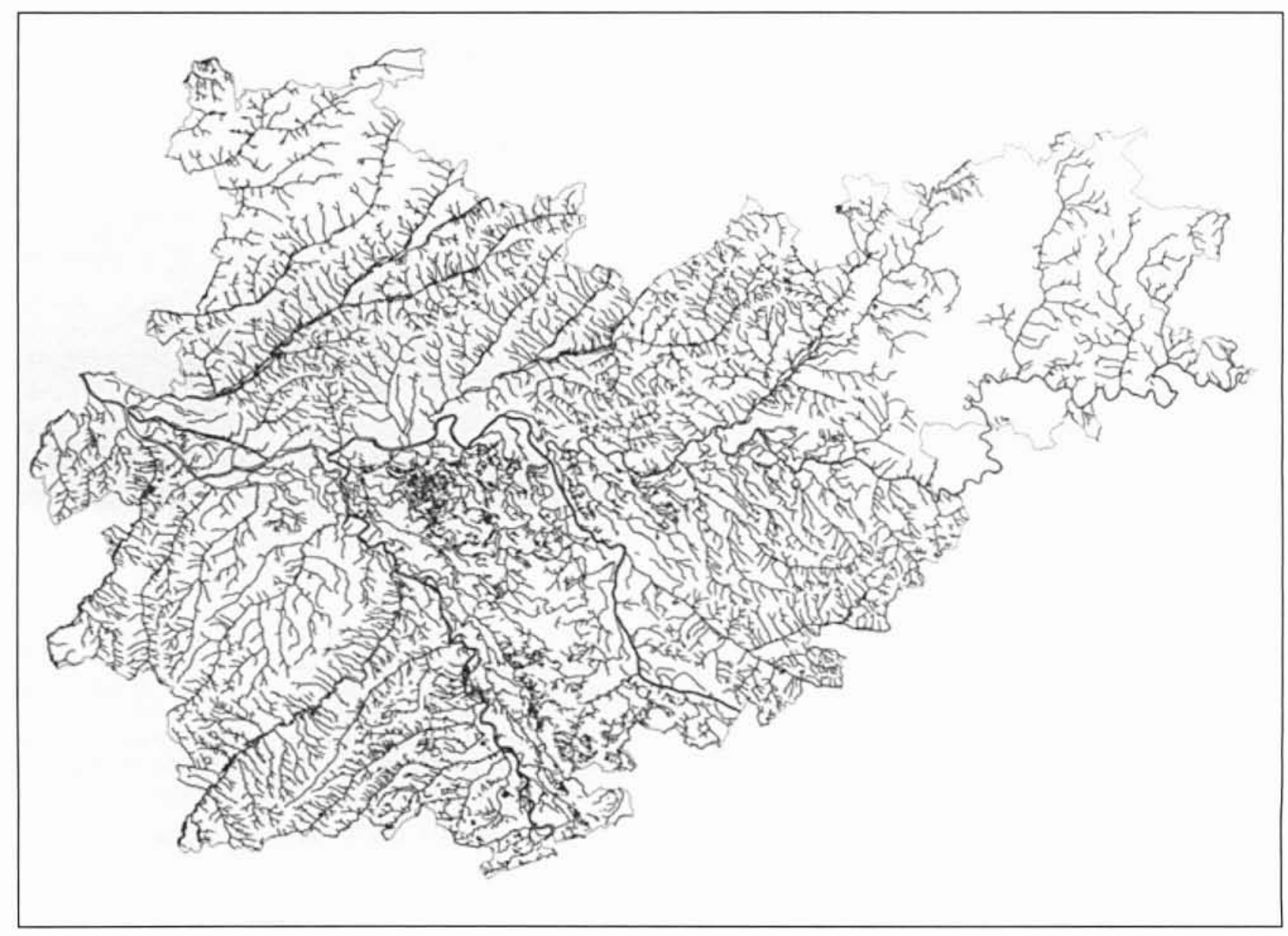

3. Réseau hydrographique. 


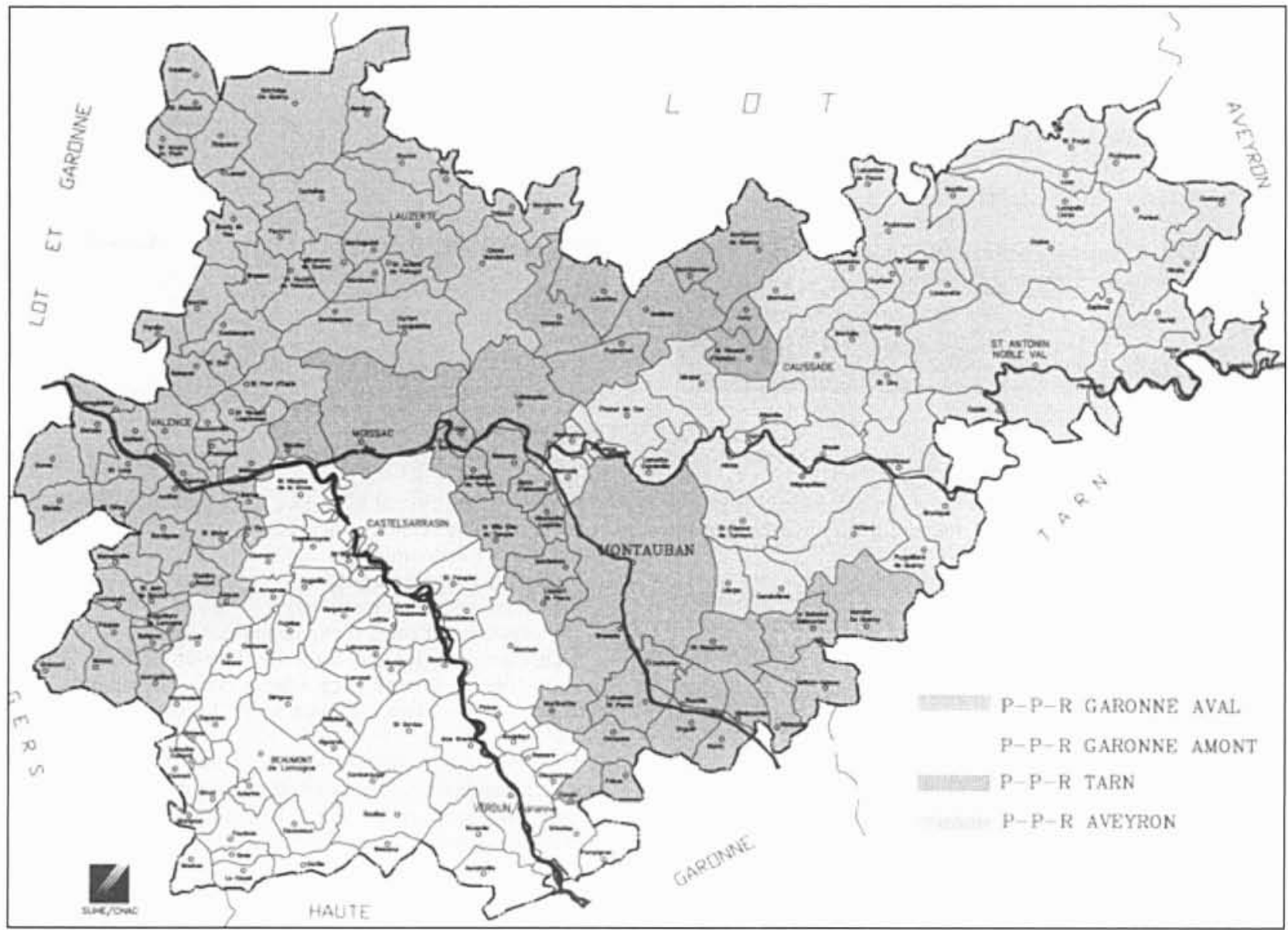

\section{Carte des 4 secteurs PPR.}

Dans les centres anciens historiques denses couverts par un service d'annonce des crues, la vie des quartiers est maintenue sans augmenter la population soumise aux risques

A Montauban et à Moissac, 2 zones d'activité protégées par des digues contre une crue centennale accueilleront, sous conditions, des E.R.P (Etablissements recevant du Public). non sensibles.

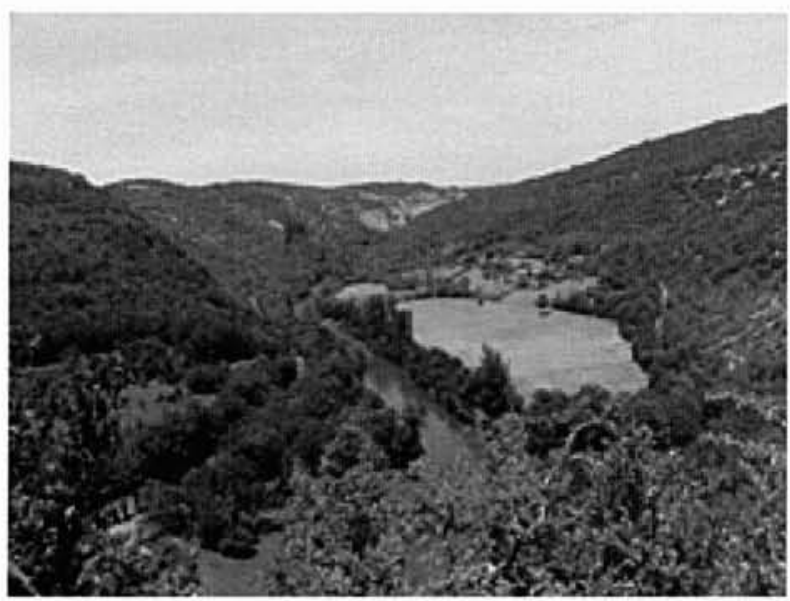

L'Aveyron à St-Antonin

\subsection{Difficultés rencontrées}

La première difficulté a été de faire référence à la crue historique dans un département où la majorité des documents approuvés prenait en compte la crue centennale. Elle s'est progressivement estompée grâce à la campagne de concertation menée tant par le Préfet que par la DDE.

La deuxième a concerné l'urbanisation de 2 zones nouvelles d'activité protégées par des digues contre une crue

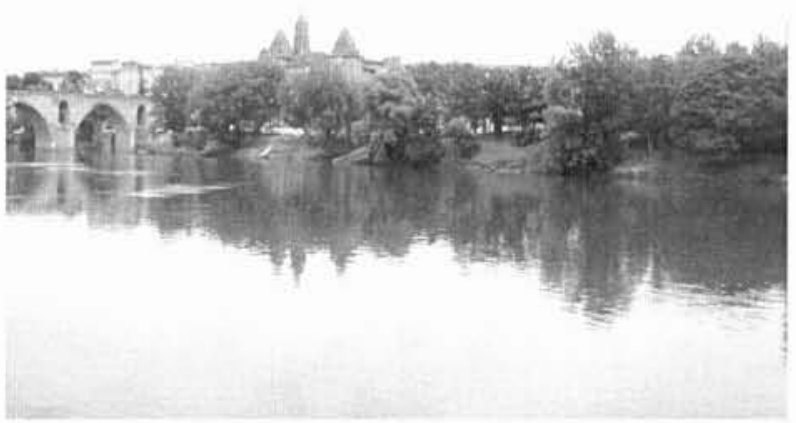

Le Tarn à Montauban 


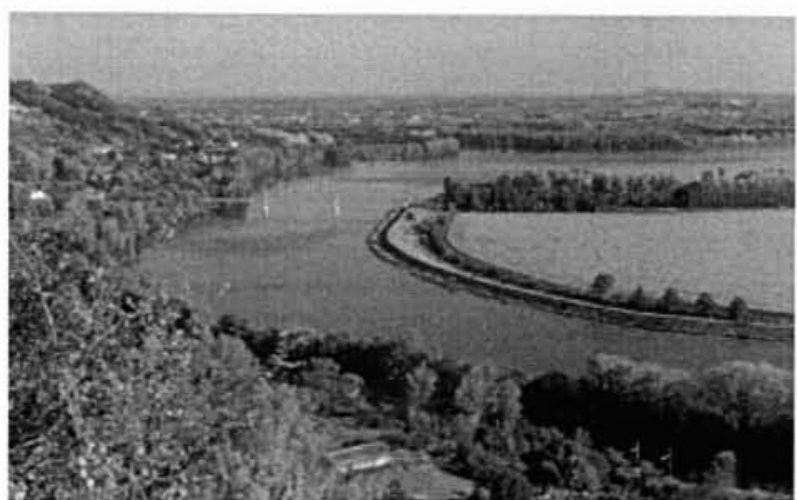

La Garonne

centennale, qui a motivé une inspection mandatée par le ministère de l'Environnement.

\subsection{Suites envisagées}

L'Etat "régalien" a mené une politique ambitieuse de reconquête des champs d'expansion des crues et en l'espace de 3 ans le bon sens a été retrouvé. à :

Il va permettre à l'Etat "partenaire" d'aider les communes

- repenser un développement moins consommateur d'espace (révision des POS, opérations remembrement-aménagement),

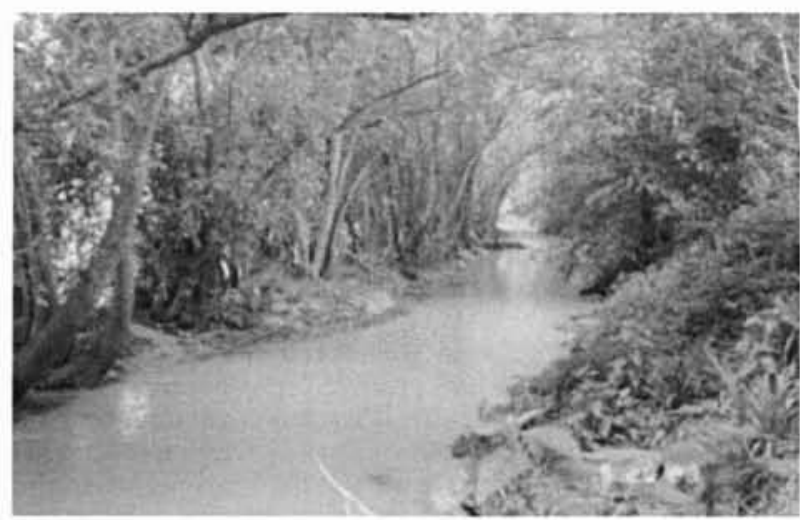

\section{Petit ruisseau}

- réhabiliter les centres anciens,

- protéger l'économie agricole,

- requalifier les paysages,

- restaurer les zones humides,

- valoriser les champs d'expansion des crues.

Une étude sur les paysages de Tarn-et-Garonne pilotée par un pôle de compétence Etat servira de support à ces actions pour favoriser à moyen terme le tourisme autour de l'eau.

La cartographie informatique en cours d'élaboration qui touchera chaque citoyen, permettra sans doute à chacun de comprendre enfin que l'eau est incompressible et que les événements naturels ne peuvent que difficilement être totalement maîtrisés par l'homme. 